\title{
Smad4 re-expression increases the sensitivity to parthenolide in colorectal cancer
}

\author{
XUEMEI LI, HUIKE YANG, JIA KE, BAOQUAN LIU, XIAOHONG LV, XINLEI LI and YAFANG ZHANG \\ Department of Anatomy, Harbin Medical University, Harbin, Heilongjiang 150086, P.R. China
}

Received January 25, 2017; Accepted August 8, 2017

DOI: $10.3892 /$ or.2017.5929

\begin{abstract}
Parthenolide (PT), a sesquiterpene lactone extracted from the plant feverfew, has been demonstrated to have anti-inflammatory and anticancer properties. Although PT has been revealed to markedly inhibit colorectal cancer cell proliferation, the inhibitory effects decrease with administration time. These findings revealed that colorectal cancer cells develop resistance to PT. However, the underlying mechanism is unclear. In the present study we observed significantly low expression of Smad4 in 3 PT-resistant cell lines (HCT-116/PT, HT-29/PT and Caco-2/PT), which were obtained using in vitro concentration gradient-increased induction, but not in their parental cells. In the present study we used the lentiviral-mediated transfection method to upregulate Smad4 in resistant colorectal cancer cell lines. Flow cytometry assay was used to assess cell apoptosis. Cell migration was detected using a QCM $^{\text {TM }}$ 24-well Fluorimetric Cell Migration Assay kit. Our study showed that Smad4 overexpression notably decreased the half maximal inhibitory concentration $\left(\mathrm{IC}_{50}\right)$ values for PT in the 3 PT-resistant cell lines, and improved the inhibitory effects of PT on cell migration and enhanced apoptosis in vitro as well as suppressed xenografted tumors in a PT-resistant colorectal cancer mouse model. Further study by western blotting into the underlying mechanism demonstrated that Smad4 overexpression suppressed the expression of MDR1 in the resistant cells, and resulted in the accumulation of PT, which in turn promoted the expession of caspase- 3 and Bax and inhibited the expression of $\mathrm{Bcl}-2$ and the phosphorylation of NF- $\kappa \mathrm{B}$ p65. In short, Smad4 re-expression may be crucial for enhancing the sensitivity and reversing the resistance to PT in PT-resistant colorectal cancer cells.
\end{abstract}

Correspondence to: Dr Yafang Zhang, Department of Anatomy, Harbin Medical University, 194 Xuefu Road, Harbin, Heilongjiang 150086, P.R. China

E-mail: yafangzhang2008@aliyun.com

Key words: parthenolide, colorectal cancer, drug resistance, Smad4

\section{Introduction}

In addition to its anti-inflammatory and analgesic actions, the antitumor function of parthenolide (PT), a sesquiterpene lactone extracted from the plant feverfew, has been widely studied in recent years. For example, Sun et al demonstrated that PT inhibited proliferation and induced autophagy in HepG2 cells by suppressing the expression of Ki67, a proliferation-related gene. Moreover, PT has been demonstrated to promote apoptosis in hepatoma carcinoma cells by downregulating Bcl-2 and upregulating p53 and Bax (1). PT also decreased the viability of MDA-MB-231 breast cancer cells. Inhibition of MDA-MB-231 cells by PT may be associated with superoxide anion and highly reactive oxygen species (ROS) (2). Studies have also revealed that PT inhibited SiHa and MCF-7 breast cancer cells in a concentration-dependent manner by upregulating p53, Bax, caspase-3 and -6 and inhibiting Bcl-2 (3). PT increased ROS, and then induced cell death, AMPK activation and the cell cycle arrest in breast cancer cells (4). In addition, Yu et al reported that in oral cancer cells, PT-induced apoptosis was impaired by siRNA targeting Bcl-2-interacting mediator of cell death (BIM). PT also increased the expression of death receptor 5 (DR5), which is closely related to caspase8-mediated apoptosis. Thus, PT promoted BIM and DR5 expression and subsequently induced apoptosis in oral cancer cells to suppress cancer (5). Collectively, these studies revealed that PT inhibits various tumor cells and solid tumors.

We revealed that PT markedly inhibited the proliferation and migration of several colorectal cancer cell lines. However, the half maximal inhibitory concentration $\left(\mathrm{IC}_{50}\right)$ of $\mathrm{PT}$ in colorectal cancer cells increased during long-term administration, indicating the development of PT resistance. As few studies have examined the mechanism underlying PT resistance, we focused on this resistance in the present study. To this end, we selected 3 colorectal cancer cell lines, HCT-116, HT-29 and Caco-2, to establish PT-resistant cell lines using the concentration gradient-increased induction method and screened for differentially expressed genes in the resistant cells and their parental cells using gene arrays. Bioinformatic analysis and data summary significantly revealed lower expression of Smad4 in the resistant cells than in the parental cells, which was confirmed by subsequent evaluation of Smad4 mRNA and protein levels in the resistant cells and parental cells (data not shown). The literature search revealed that Smad4 has a direct effect on the phosphorylation, or the 
nuclear transport of Smad2 and Smad3, thus, the change in the expression of Smad4 may affect downstream proteins in the TGF- $\beta$ signaling pathway. For these reasons, we decided to select Smad4 as the target for the present study.

Smad4 is closely associated with cancer: it primarily acts as a tumor suppressor and is often inactivated in tumors. Specifically, inactivation or mutation of Smad4 has been identified during the progression and metastasis of many types of cancer, including prostate (6), ovarian (7), colorectal (8) and thyroid (9) cancers. Studies of aberrant Smad4 expression and tumor drug resistance have demonstrated that Smad4 expression affects the chemosensitivity of some tumors to certain drugs. For example, Boulay et al reported that patients with colorectal cancer and normal Smad4 diploidy derived a 3 -fold higher benefit from 5-fluorouracil (5-FU)-based adjuvant chemotherapy (overall survival, 3.23, $\mathrm{P}=0.056$; disease-free survival, $2.89, \mathrm{P}=0.045$ ) than patients with a Smad4 deletion (10). Furthermore, Smad4 deletion or inactivation promoted colorectal cancer cell proliferation, migration, invasion and resistance to 5-FU, whereas LY294002, an inhibitor of the PI3K/AKT pathway, restored sensitivity to 5-FU in Smad4-deficient cells. Smad4 inactivation induced resistance to 5-FU in HCT-116 Smad4 ${ }^{-/-}$ cells (11). These studies all demonstrated the impact of Smad4 on the sensitivity of colorectal cancer cells to 5-FU. In the present study, we explored the influence of the Smad4 gene on the sensitivity to PT and the possible involvement of Smad4 target proteins in drug resistance. Moreover, we investigated the efficacy of combined Smad4 gene intervention and PT in the inhibition of the development of PT-resistant human colorectal xenografted tumors.

\section{Materials and methods}

Cell culture. Three colorectal cancer cell lines, HCT-116, HT-29 and Caco-2, were purchased from the Cell Bank of the Chinese Academy of Sciences (CCAS; Shanghai, China) and maintained in RPMI-1640 medium containing $10 \%$ fetal bovine serum (FBS) (both from Invitrogen, Shanghai, China). PT (10 $\mu \mathrm{M})$ was added to the complete medium used in the culture of HCT-116/ PT, HT-29/PT and Caco-2/PT. The viral packaging cell line 293TN was purchased from the American Type Culture Collection (ATCC; Rockville, MD, USA) and maintained in Dulbecco's minimum essential medium, (DMEM; Invitrogen) supplemented with $10 \%$ FBS. All cells were incubated at $37^{\circ} \mathrm{C}$ in a humidified $95 \%$ air and $5 \% \mathrm{CO}_{2}$ incubator. All adherent cells were passaged using $0.25 \%$ trypsin (Invitrogen) digestion.

Establishment of resistant colorectal cancer cell lines by induction. HCT-116, HT-29 and Caco-2 cells were maintained according to routine protocols, except for the addition of PT (starting at $0.5 \mu \mathrm{M}$ ) (Sigma-Aldrich, Carlsbad, CA, USA) to the medium; the concentration of PT was doubled every 3 passages, i.e., 1, 2, 4, 8, 16 and $32 \mu \mathrm{M}$. After being cultured in medium containing $32 \mu \mathrm{M}$ PT for 3 passages, resistant cells were named as HCT-116/PT, HT-29/PT and Caco-2/PT. We then examined the $\mathrm{IC}_{50}$ values for PT in the resistant cell lines and their parental cells. The resistant cells as well as their parental cell lines were seeded into 96-well plates (Corning, Corning, NY, USA) at a concentration of $1 \times 10^{5}$ cells/well and cultured for $48 \mathrm{~h}$ in medium containing
PT at final concentrations of $1,2,4,8,16$ or $32 \mu \mathrm{M}$. The Cell Counting Kit-8 (CCK-8; Dojindo, Kumamoto, Japan) assay was employed to assess the sensitivity of the cells to PT, and the $\mathrm{IC}_{50}$ values were calculated. Moreover, PT-resistant cell lines and their parental cell lines $\left(1 \times 10^{6}\right.$ cells) were harvested for total RNA and protein extraction, followed by real-time PCR and western blotting for Smad4 assessment.

Construction of a pcDNA-Smad4 plasmid. The coding sequence (CDS) of human Smad4 (NM_005359.5) was amplified using the primers 5'-GGAATTCGCCACCATGGACAA TGT GTCTATTACG-3' and 5'-CGGGATCCTCAGTCTAAA GGTTGTGGGTCTG-3', which contained an EcoRI site, a Kozak sequence and a BamHI site. cDNA was prepared by reverse transcription using RNA isolated from 293TN cells. The PCR product was digested and cloned into a pcDH1-CMV lentiviral expression vector (System Biosciences, Mountain View, CA, USA); the recombinant vector was named pcDH1Smad4. The construct integrity was confirmed by DNA sequencing, and endotoxin-free DNA was prepared in all cases. The products of the vectors were confirmed by DNA sequencing, and endotoxin-free DNA was prepared.

Lentivirus packaging. One day prior to transfection, 293TN cells were seeded into $10-\mathrm{cm}$ dishes and co-transfected with $2 \mu \mathrm{g}$ of the pcDH1-Smad4 vector and $10 \mu \mathrm{g}$ of pPACK Packaging Plasmid mix (System Biosciences) using Lipofectamine 2000 (Invitrogen) in accordance with the manufacturer's protocol. The medium was then replaced with DMEM containing 1\% FBS. After $48 \mathrm{~h}$, the supernatant was harvested and then cleared by centrifugation for $5 \mathrm{~min}$ at $5,000 \mathrm{x}$ g and $4^{\circ} \mathrm{C}$ before being passed through a $0.45-\mu \mathrm{m}$ polyvinylidene fluoride (PVDF) membrane (Millipore, Billerica, MA, USA). The viral titer was determined by gradient dilution, and the packaged lentivirus was named Lv-Smad4. A control virus carrying no insert gene, Lv-control, was constructed as well.

Overexpression of Smad4 in PT-resistant colorectal cancer cell lines using a lentiviral approach. HCT-116/PT, HT-29/PT and Caco-2/PT cells in logarithmic growth phase were seeded into 6 -well plates at $5 \times 10^{5}$ cells/well, and cultured for $24 \mathrm{~h}$ in complete medium at $37^{\circ} \mathrm{C}$ and $5 \% \mathrm{CO}_{2}$. One day later, a viral solution (Lv-control or Lv-Smad4) was added at a multiplicity of infection (MOI) of 10. The infection efficiency was observed using a fluorescent marker at $72 \mathrm{~h}$ after infection. Total RNA and protein were isolated from the cells and subjected to real-time PCR and western blotting for evaluation of Smad4 mRNA and protein levels, respectively.

Assessment of cell viability and $I C_{50}$ values. The 3 cell lines and their derivatives were seeded into 96-well plates at $5 \times 10^{4}$ cells/well and cultured in medium containing PT at final concentrations of $1,2,4,8,16$ or $32 \mu \mathrm{M}$ for $48 \mathrm{~h}$, followed by a CCK-8 assay to assess cell viability. Briefly, $10 \mu \mathrm{l}$ of CCK-8 solution was added, and the cells were then cultured under normal conditions for an additional $4 \mathrm{~h}$ before measuring the absorbance at $450 \mathrm{~nm}$. The inhibition ratio was calculated, and the fitted equation between the inhibition ratio and PT concentration was obtained for computation of the $\mathrm{IC}_{50}$ values for PT at $48 \mathrm{~h}$. 
Detection of apoptosis. The resistant cells were seeded into 6 -well plates at $1 \times 10^{5}$ cells/well in medium containing $10 \mu \mathrm{M}$ PT and cultured for $48 \mathrm{~h}$. The cells were collected, and apoptosis was assessed using flow cytometry (FACSCalibur) after treatment with Annexin V/FITC apoptosis detection kit II (cat. 556570) (both from BD Biosciences, San Jose, CA, USA). Each cell line was grouped as control cells, cells infected with Lv-control and cells infected with Lv-Smad4, and then cultured in the presence or absence of PT. For lentiviral infection, cells were employed in the experiment $72 \mathrm{~h}$ after infection. The experiments were carried out in 3 resistant cell lines.

Assessment of cell migration. Cell migration was assessed using a QCM $^{\mathrm{TM}}$ 24-well Fluorimetric Cell Migration Assay kit (Chemicon, Temecula, CA, USA) in accordance with the manufacturer's instructions. Cells were seeded into the upper chamber at $1 \times 10^{6}$ cells/well, and $500 \mu \mathrm{l}$ of complete medium containing $10 \%$ FBS was added to each well in the lower chamber. Cells that had migrated to the underside of the membrane were fixed in $4 \%$ paraformaldehyde after $48 \mathrm{~h}$. The invading cells were stained with DAPI, and fluorescence was quantified. Data are reported as relative fluorescence units (RFUs). The grouping was the same as that in the apoptosis assay.

Effects of overexpression of Smad4 on relevant proteins in PT-resistant colorectal cancer cells. HCT-116/PT, HT-29/PT and Caco-2/PT and those infected with Lv-Smad4 for $72 \mathrm{~h}$ were seeded into 6 -well plates at $1 \times 10^{5}$ cells/well and treated with PT $(10 \mu \mathrm{M})$ or without PT. After being cultured at $37^{\circ} \mathrm{C}$ under $5 \% \mathrm{CO}_{2}$ for $48 \mathrm{~h}$, the cells were harvested, and total protein was extracted for western blotting to assess the levels of Smad4, MDR1, Bcl-2, Bax and caspase-3, as well as the phosphorylation of NF- $\kappa \mathrm{B}$ p65.

Combination for subcutaneous tumor inhibition. Six-week-old male BALB/c nude mice, provided by the Animal Experiment Center of the Second Military Medical University, were randomly divided into 3 groups: the model group (16 mice, inoculated with Caco-2/PT), the Lv-control group (8 mice, inoculated with Caco-2/PT infected with Lv-control) and the Smad4 overexpression group (8 mice, inoculated with $\mathrm{Caco}-2 / \mathrm{PT}$ infected with Lv-Smad4). Suspensions of Caco-2/PT, Caco-2/PT + Lv-control or Caco-2/PT $+\mathrm{Lv}-\mathrm{Smad} 4\left(1 \times 10^{7}\right.$ cells $)$ cells were prepared in $50 \mu \mathrm{l}$ of dPBS and subcutaneously injected into the left foreflank of each mouse; tumors formed within 15 days. The model group was divided into treated and untreated groups. The treated, $\mathrm{Lv}$-control and Smad4 overexpression groups were administered daily with $10 \mathrm{mg} / \mathrm{kg}$ PT [200 ng/ $\mu \mathrm{l}$, prepared in normal saline from a $20 \mu \mathrm{g} / \mu \mathrm{l}$ stock solution prepared with dimethyl sulfoxide (DMSO)] via caudal vein injection, and the untreated group was injected with normal saline. The treatment period was 5 weeks. The tumor diameter was assessed every week, and the tumor volumes were calculated. All mouse experiments were approved by the Medical Ethics Review Committee of Harbin Medical University.

Assessment of mRNA levels. Total RNA was isolated using TRIzol reagent (Invitrogen) according to the manufacturer's instruction and reverse-transcribed into cDNA using M-MLV reverse transcriptase and an oligo(dT)18 primer (both from
Takara, Shiga, Japan). The following specific primers were used for quantitative PCR of human Smad4 and $\beta$-actin to generate amplified products of 222 and $211 \mathrm{bp}$, respectively: Smad4, 5'-ACCACCAAAACGGCCATCTTCAG-3' and 5'-GGTCCACGTATCCATCAACAGTA-3'; $\beta$-actin, 5'-CCT GTACGCCAACACAGTGC-3' and 5'-ATACTCCTGCTTGC ATCC-3'. Real-time PCR was performed using a SYBR ${ }^{\circledR}$ Premix Ex Taq ${ }^{\mathrm{TM}}$ kit and a TP800 system (both from Takara). cDNA from $200 \mathrm{ng}$ of total RNA was used as the template. The PCR reactions were carried out under the following conditions: 40 cycles of denaturation at $95^{\circ} \mathrm{C}$ for $10 \mathrm{sec}$, annealing at $60^{\circ} \mathrm{C}$ for $20 \mathrm{sec}$ and extension at $72^{\circ} \mathrm{C}$ for $20 \mathrm{sec}$. The mRNA levels of Smad4 were normalized to those of an endogenous housekeeping gene, $\beta$-actin, using the $\Delta \Delta \mathrm{Ct}$ method.

Assessment of protein levels. Total protein was extracted from cells using M-PER mammalian protein extraction reagent (Pierce, Rockford, IL, USA). Equal amounts of protein (25 $\mu \mathrm{g} /$ lane), as estimated using the bicinchoninic acid (BCA) protein assay (Pierce), were separated by $11 \%$ sodium dodecyl sulfate-polyacrylamide gel electrophoresis (SDS-PAGE) and transferred onto nitrocellulose membranes. The blots were probed with a monoclonal antibody against human Smad4 (1:1,000), anti-MDR1 (1:500), anti-p-p65/p65 (1:500), anti-Bcl-2 (1:200), anti-Bax (1:500), anti-caspase-3 (1:300) and anti- $\beta$-actin (1:800), followed by a secondary horseradish peroxidase (HRP)-conjugated anti-mouse/rabbit antibody (Santa Cruz Biotechnology, Santa Cruz, CA, USA). After being washed, the bands were detected using chemiluminescence and exposed to X-ray films. $\beta$-actin was used as an endogenous reference for normalization.

Statistical analysis. All data are expressed as the mean \pm standard deviation (SD). Factorial analysis was employed for inter- and intra-group comparisons. Statistical significance was determined by Student's t-test for comparison of two groups, and means were compared with a Chi-square test. P-values $<0.05$ were considered to be statistically significant. All statistical analyses were performed using SPSS 13.0 software (SPSS, Inc., Chicago, IL, USA).

\section{Results}

Assessment of Smad4 $m R N A$ and protein. The real-time PCR and western blotting results for Smad4 in 3 PT-resistant cell lines revealed decreases at both the transcriptional level (Fig. 1A-a, B-a and C-a) and protein level (Fig. 1A-b, B-b and $\mathrm{C}-\mathrm{b})$. There was a significant difference in PT-resistant cells and parental cells $(\mathrm{P}<0.01)$. Lentiviral-mediated overexpression of Smad4 in these 3 PT-resistant cell lines resulted in a gene delivery efficiency close to $100 \%$ at $72 \mathrm{~h}$ after infection, as demonstrated by green fluorescent protein (GFP) expression (Fig. 1A-c, B-c and C-c). Furthermore, protein assessments revealed that the protein levels of Smad4 (Fig. 1A-d, B-d and C-d) were significantly increased in the groups infected with $\mathrm{Lv}-\mathrm{Smad} 4$ compared with the control cells or the Lv-control group $(\mathrm{P}<0.01)$, and there was no significant difference in the control cells or the LV-control group $(\mathrm{P}>0.05)$, indicating that the lentiviral system effectively delivered exogenous Smad4 gene in these cells. 
A a

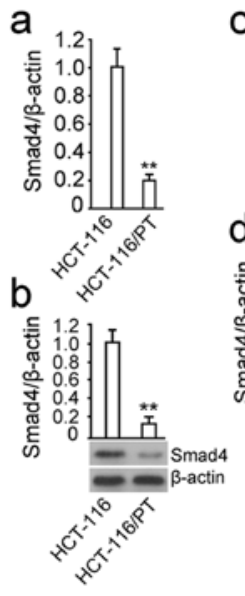

C

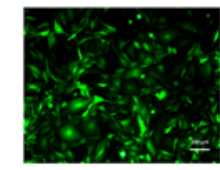

d

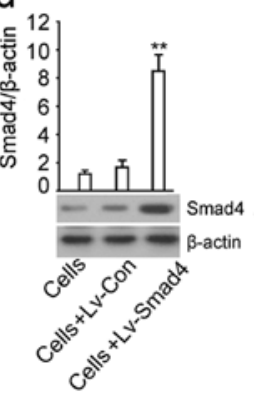

C a

b
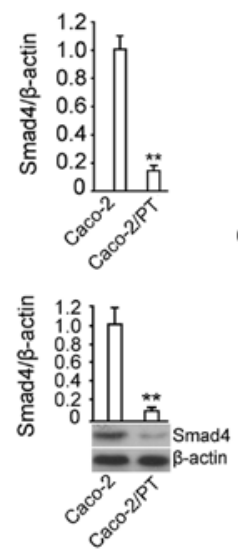

B a
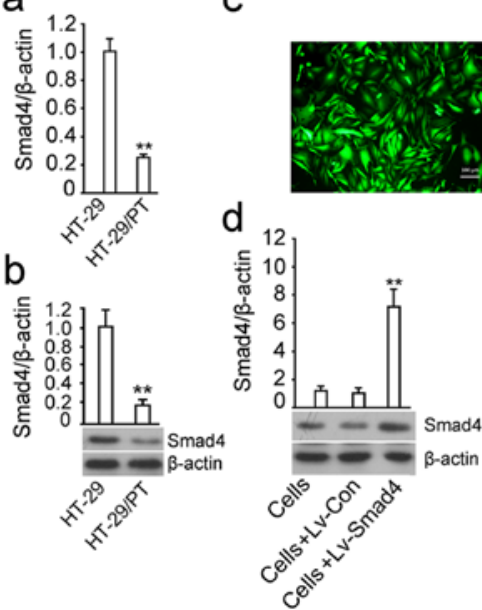

C

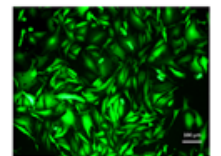

C
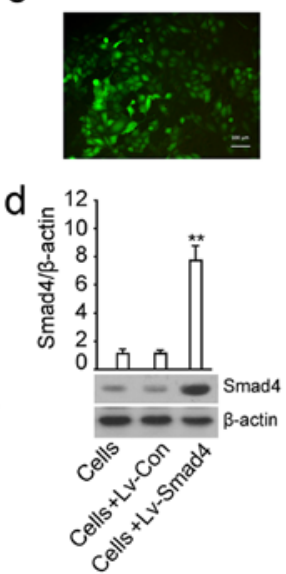

Figure 1. Assessment of Smad4 mRNA and protein. The mRNA and protein levels of Smad4 in the PT-resistant cells and their parental cells were assessed by (A-a, B-a and C-a) real-time PCR and (A-b, B-b and C-b) western blotting, respectively. (A-c, B-c and C-c) Cells were infected with lentivirus at an MOI of 10 and photographed $72 \mathrm{~h}$ after infection at a magnification power of $\mathrm{x} 200$. The ratio of the number of cells expressing GFP to the number of total cells/field was used to calculate the gene delivery rate. (A-d, B-d and C-d) The protein levels of Smad4 in PT-resistant cells uninfected and infected with Lv-control and $\mathrm{Lv}$-Smad4 were assessed by western blotting. The results are expressed as the mean $\pm \mathrm{SD}$ of at least 3 separate experiments; ${ }^{* *} \mathrm{P}<0.01$ compared to the Lv-control group.
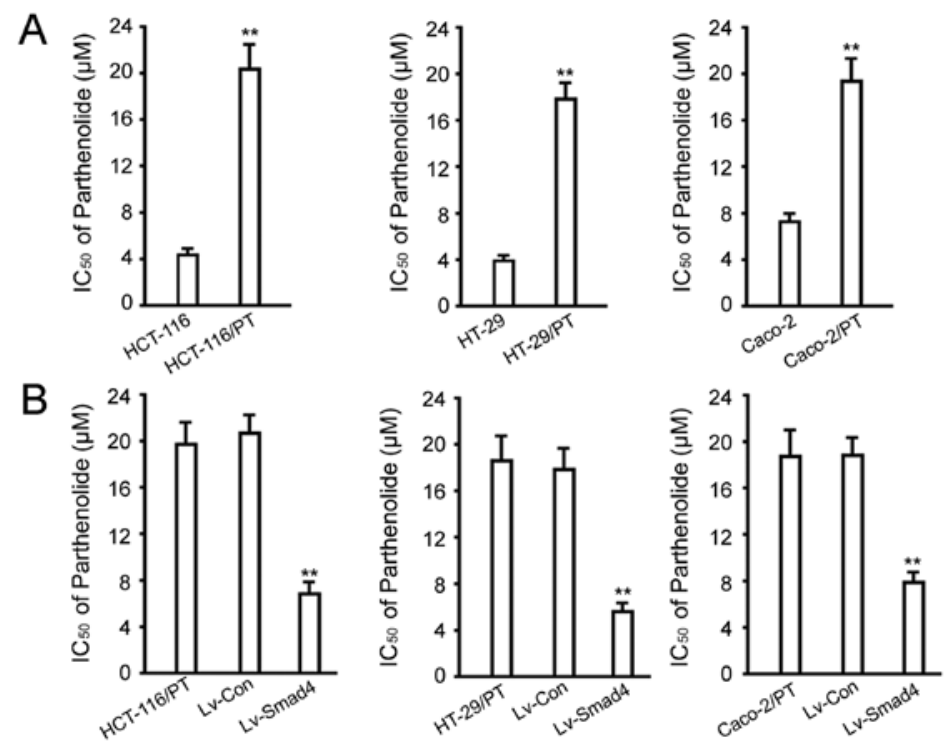

Figure 2. $\mathrm{IC}_{50}$ value analysis. (A) $\mathrm{IC}_{50}$ value analysis of PT-resistant cells and respective parental cells. The left, middle and right panels are the $\mathrm{IC}_{50}$ values of PT at $48 \mathrm{~h}$ in PT-resistant HCT-116, HT-29 and Caco-2 cells, respectively, as well as their respective parental cells. (B) IC $_{50}$ analysis of PT-resistant cells before and after overexpression of Smad4. The left, middle and right panels show the $\mathrm{IC}_{50}$ values of PT at $48 \mathrm{~h}$ in PT-resistant HCT-116, HT-29 and Caco-2 cells, respectively, and the respective cells infected with Lv-control and Lv-Smad4. The results are expressed as the mean \pm SD of at least 3 separate experiments. ${ }^{* *} \mathrm{P}<0.01$ compared to the $\mathrm{Lv}$-control group. 
A

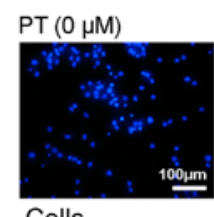

PT $(10 \mu \mathrm{M})$
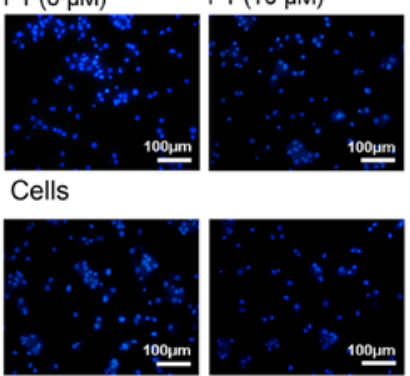

Cells infected with Lv-Con
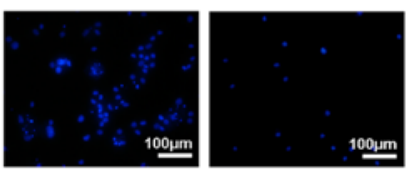

Cells infected with Lv-Smad4

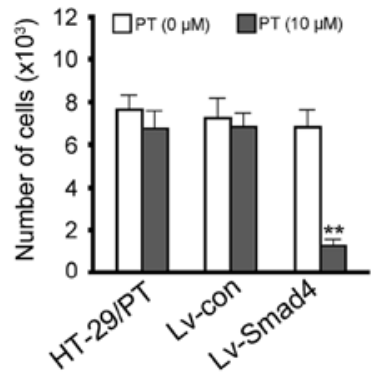

B

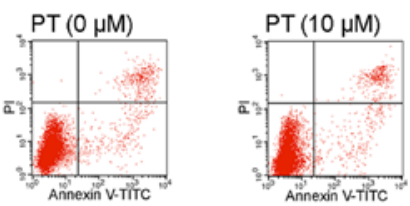
Cells

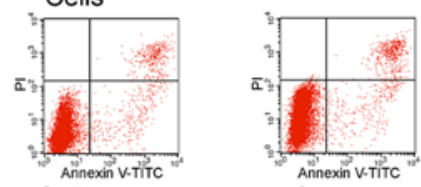

Cells infected with Lv-Con

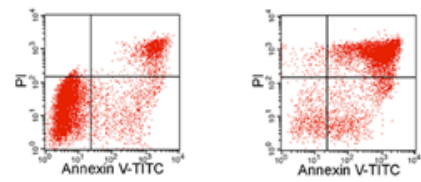

Cells infected with Lv-Smad4

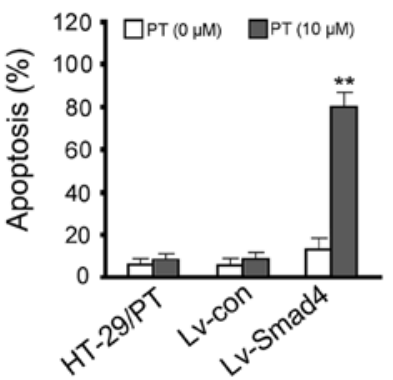

Figure 3. Assessment of cell migration and apoptosis. (A) The invading cells were stained with DAPI. The upper image shows cells at a power of x200; the blue stain shows the nuclei of cells traversing the membrane. Fluorescence was then quantified, and the results are reported as relative fluorescence units (RFUs). The $\mathrm{x}$-coordinate represents the experimental groups, and the $\mathrm{y}$-coordinate is the number of cells that traversed the membrane (lower image). (B) The cells were treated with $10 \mu \mathrm{M}$ PT for $48 \mathrm{~h}$, and apoptosis was analyzed by flow cytometry. The upper image shows representative dot plots; the x-coordinate is the FITC channel, and the y-coordinate shows PI staining. The lower left panel represents living cells, the lower right panel shows early apoptotic cells, the upper left panel shows necrotic cells, and the upper right panel shows late apoptotic cells. The lower image shows the data analysis; the x-coordinate represents the experimental groups, and the $\mathrm{y}$-coordinate represents the apoptosis rate (including early and late apoptotic cells). The results are expressed as the mean \pm SD of at least 3 separate experiments; ${ }^{* *} \mathrm{P}<0.01$ compared to the HT-29/PT or HT-29/PT infected with Lv-control group.

$I C_{50}$ value analysis. We analyzed the $\mathrm{IC}_{50}$ values to ascertain the successful establishment of PT-resistant cell lines. As expected, the $\mathrm{IC}_{50}$ values of the HCT-116/PT, HT-29/PT and Caco-2/PT cell lines increased from $4.09 \pm 0.76,3.97 \pm 0.58$ and $7.13 \pm 1.21 \mu \mathrm{M}$ to $20.31 \pm 3.21,17.65 \pm 1.89$ and $18.64 \pm 3.02 \mu \mathrm{M}$ after $48 \mathrm{~h}$, respectively ( $\mathrm{P}<0.01$, vs. the parental cells; Fig. 2A). Overexpression of Smad4 in HCT-116/PT, HT-29/PT and Caco-2/PT cells significantly decreased the $\mathrm{IC}_{50}$ values to $6.12 \pm 2.11,5.75 \pm 1.08$ and $7.86 \pm 1.95 \mu \mathrm{M}(\mathrm{P}<0.01$, vs. the control cells or the Lv-control group; Fig. 2B), respectively.

Assessment of cell migration and apoptosis. We employed a Transwell experiment to explore changes in tumor cell migration, which plays an important role in tumor metastasis. In the absence of PT, there was no statistical difference in the number of cells penetrating the basal membrane between the Lv-Smad4 and the control cells or the Lv-control group ( $\mathrm{P}>0.05)$; in the presence of $10 \mu \mathrm{M} \mathrm{PT}$, overexpression of Smad4 decreased the number of cells that penetrated the basement membrane $(\mathrm{P}<0.01$, vs. the control cells or the $\mathrm{Lv}$-control group; Fig. 3A), and there was no statistical difference between the control cell group and Lv-control group $(\mathrm{P}>0.05)$. The data also revealed that treatment with $10 \mu \mathrm{M}$ PT had no significant impact on HT-29 and HT-29 infected with Lv-control (P>0.05), however it significantly inhibited the migration in HT-29

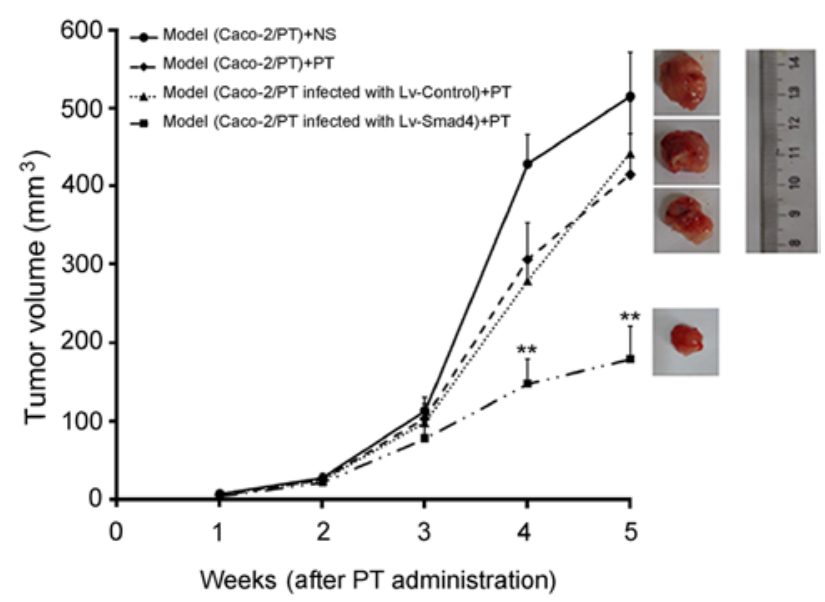

Figure 4. In vivo tumor inhibition experiment. The inhibition of tumor formation in vivo by a combination of Smad4 overexpression and PT. The $\mathrm{x}$-coordinate is the time of PT administration; administration began 16 days after tumor inoculation. The y-coordinate is the volume of the tumor $(\mathrm{V}=\mathrm{ab} / 2$, where $\mathrm{a}$ and $\mathrm{b}$ are the measured long and short diameters of the tumor). Each group included 8 animals, and the long and short diameters of each sample were measured 3 times; the mean value was used for statistical analysis; ${ }^{* *} \mathrm{P}<0.01$ compared to the $\mathrm{PT}$ administration group.

cells infected with Lv-Smad4 $(\mathrm{P}<0.05$, PT group vs. non-PT group;Fig. 3A). The results indicate that overexpression of 

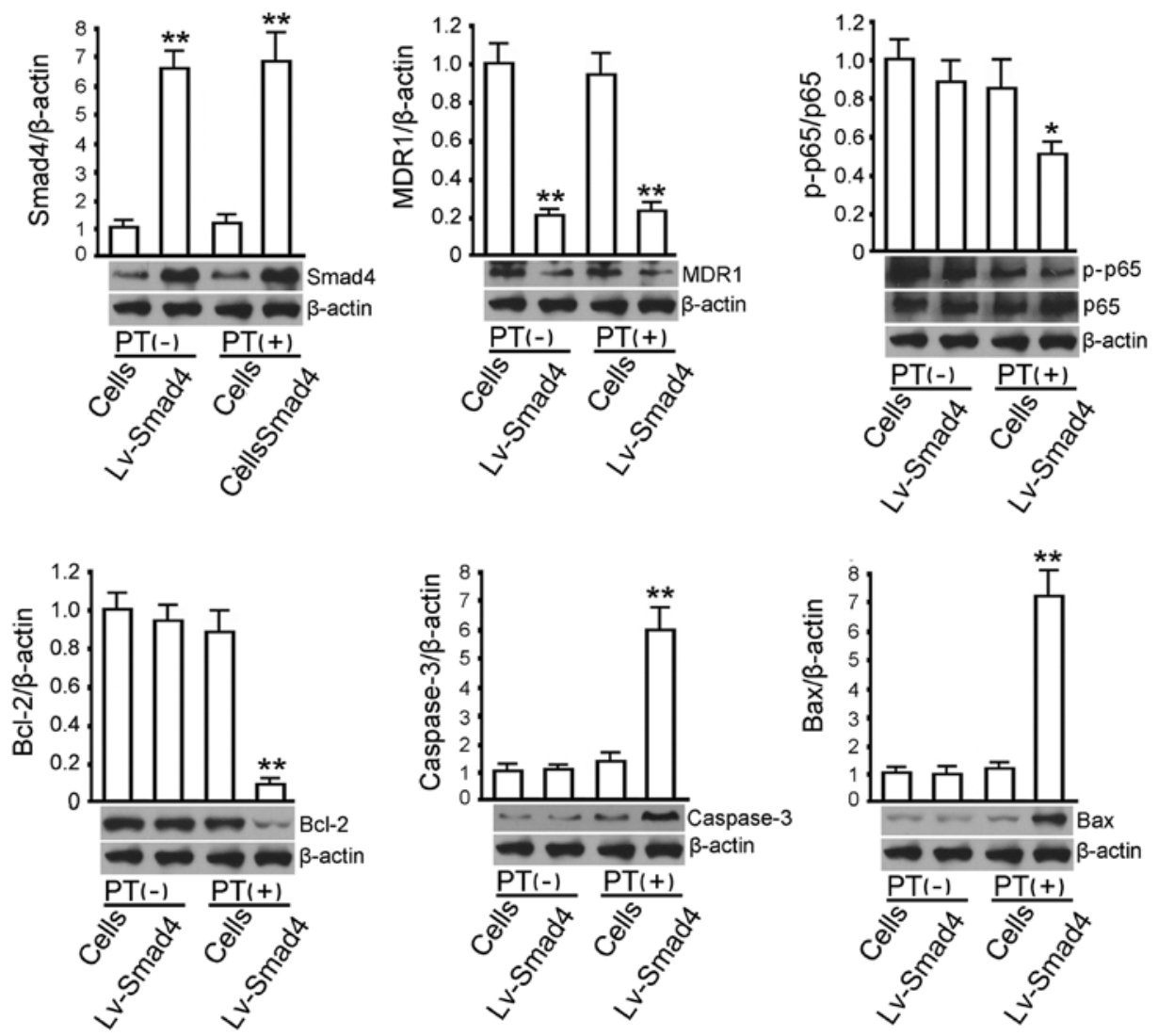

Figure 5. Assessment of protein expression. Changes in relevant proteins before and after overexpression of Smad4 in the Caco-2/PT cells. Effect of Smad4 on relevant proteins in the presence or absence of PT. The lower figure is the scanned image of an X-ray film for assessing protein expression, and the upper figure is an analysis of the differences in 6 proteins between groups. The x-coordinate represents the experimental groups, and the y-coordinate represents the relative protein content. $\beta$-actin was used as the reference protein, and total p65 was used as the reference for the determination of phosphorylated p65. The results are expressed as the mean $\pm \mathrm{SD}$ of at least 3 separate experiments; ${ }^{*} \mathrm{P}<0.05$ and ${ }^{* *} \mathrm{P}<0.01$ compared to the cell group.

Smad4 has no direct impact on the migration of HT-29/PT, but may increase its sensitivity to PT, thus an increased response to PT was observed. Consistent with the data of the migration assay, overexpression of Smad4 did not induce apoptosis in HT-29/PT cells, but it restored its sensitivity to PT, thus PT exerts its apoptosis-inducing activity (Fig. 3B). We also performed these assays in HCT-116/PT and Caco-2/PT cell lines, and observed similar results (data not shown).

Tumor inhibition experiment. The results of the in vivo tumor inhibition experiment revealed that administration of PT for 5 weeks only slightly inhibited tumor formation by Caco-2/PT cells, but significantly inhibited tumor formation by Caco-2/PT cells overexpressing Smad4 (Fig. 4). At the fifth week after administration, the tumor volumes were $535.29 \pm 80.29 \mathrm{~mm}^{3}$ in the model group and $431.40 \pm 60.00 \mathrm{~mm}^{3}$ in the PT administration group, corresponding to an inhibition rate of $19.42 \%$ and a non-significant difference. However, the tumor volume in the Smad4-overexpressing group that received PT averaged $189.75 \pm 42.18 \mathrm{~mm}^{3}$, corresponding to an inhibition rate of $64.55 \%$ and a significant difference compared to the control group $(\mathrm{P}<0.01)$. The data from the in vivo tumor inhibition experiment were in line with the overexpression of Smad4 resensitizing the 3 PT-resistant cell lines.

Analysis of the pathway involved in Smad4 overexpression-mediated increases in sensitivity to PT in resistant colorectal cancer cells. To elucidate how Smad4 overexpression increases the sensitivity of resistant colorectal cancer cells to PT, we examined a number of proteins that are related to Smad4 expression and are associated with drug resistance. In the absence of PT, the level of Smad4 in the $\mathrm{Lv}-\mathrm{Smad} 4$ group was significantly higher than that in the control cells $(\mathrm{P}<0.01$; Fig. 5). The opposite was observed with MDR1. No observable difference was found in the phosphorylation of NF- $\mathrm{BB}$ p65, and in the expression of Bcl-2, caspase- 3 and $\mathrm{Bax}(\mathrm{P}>0.05)$. When the cells were treated with $10 \mu \mathrm{M}$ PT for $48 \mathrm{~h}$, caspase- 3 and Bax in the cells infected with Lv-Smad4 were higher than in the control group $(\mathrm{P}<0.01)$, and the expression of MDR1 and $\mathrm{Bcl}-2$ and the phosphorylation of NF- $\mathrm{KB}$ p65 in the Lv-Smad4 group were lower than in the control group $(\mathrm{P}<0.05)$. The data of HCT-116/PT and HT-29/PT cell lines were in agreement with those of Caco-2/PT (data not shown).

\section{Discussion}

Recent studies have established the antitumor role of parthenolide (PT). For example, Kim et al demonstrated that PT decreases NF- $\kappa$ B p65 expression by blocking phosphorylation and subsequently degrading inhibitor of $\kappa \mathrm{B}-\alpha$ (I $\kappa \mathrm{B} \alpha$ ). PT also promotes apoptosis by downregulating Bcl-2 and Bcl-xL, two anti-apoptosis proteins, suggesting a potential therapeutic role of PT in colitis-associated colon cancer (12). Zhao et al described that PT not only triggers extrinsic 
apoptosis by upregulating TNFRSF10B and downregulating CFLAR, but also induces intrinsic apoptosis by increasing the expression of PMAIP1 and decreasing the level of MCL1 in non-small cell lung cancer (NSCLC) cells (13). In summary, these studies revealed that PT has an anticancer role that should not be ignored. Our analyses revealed that although PT inhibited proliferation in colorectal cancer cells, drug resistance developed over the course of administration, which were accompanied by changes in protein expression. The study of the relationship between PT and drug resistance has primarily focused on two aspects: i) the ability of PT to directly inhibit the proliferation of tumor cells and ii) the ability of PT to increase chemosensitivity or reverse resistance to chemotherapeutic agents. Specifically, Ghantous et al demonstrated that PT inhibits $\mathrm{JB}^{+} \mathrm{P}^{+}$cell proliferation by suppressing tumor promoter-mediated NF-KB activation (14). Furthermore, Li et al demonstrated that PT decreased levels of MMP-9, VEGF and IL-8 expression in breast cancer cells and inhibited the proliferation and migration of MDA-MB231 cells as well as the formation of lumen-like structures by human umbilical vein endothelial cells (15). Alternatively, PT has been reported to inhibit the activity of NF-KB and cell growth in 3 gastric cancer cell lines and to increase chemosensitivity (16). In particular, PT enhanced doxorubicin (DOX) aggregation and the apoptotic cytotoxicity of DOX in A549-derived doxorubicin (A549/DOX)-resistant cells and reversed DOX resistance by suppressing $\mathrm{p}$-gp expression via mechanisms that involve attenuation of $\mathrm{NF}-\kappa \mathrm{B}$ activation and HSP70 upregulation (17). Furthermore, parthenolide significantly increased 5-FU-mediated inhibition of BEL-7402/5-FU cell (hepatic carcinoma multidrug-resistant cells) proliferation and reversed the resistance of hepatic carcinoma-resistant cells (18). However, resistance to PT itself has not yet been reported. When treating colorectal cancer cells with PT, we found that long-term treatment induced resistance. Thus, we investigated the development of this resistance in colorectal cancer cell lines by examining the key proteins involved and strategies for reversing this resistance.

Smad4, which is located in the $18 \mathrm{q} 21$ region (19), is a tumor suppressor that is frequently inactivated in tumors (20). Indeed, mutation or inactivation of Smad4 not only results in a lack of TGF- $\beta 1$-induced growth inhibition (21), but also affects the chemosensitivity of cancer cells $(22,23)$. Moreover, Smad4 deficiency induced chemoresistance to 5-FU in CT26 and SW620 cells both in vitro and in vivo. Furthermore, Smad4 deficiency increased resistance to 5-FU by attenuating G1 or G2 cell cycle arrest via the PI3K/Akt/ CDC2/survivin pathway activation (24). Yu et al revealed that HDAC4 regulates Smad4 expression by inducing histone $\mathrm{H} 3$ deacetylation in the Smad4 promoter region. These authors also demonstrated that histone deacetylase 4 (HDAC4)mediated deacetylation of the Smad4 promoter may lead to 5 -FU resistance in breast cancer cells (25). These studies all revealed that $\mathrm{Smad} 4$ influences the response of tumor cells to 5-FU via different pathways. However, the effect of Smad4 on the sensitivity of tumor cells to PT has not been reported to date.

With the advance in clinical chemotherapy of colorectal cancer, many new chemotherapeutic agents have been researched, including PT (17). The present study revealed that although PT inhibited colon cancer, the cells easily developed resistance. Moreover, we revealed that Smad4 mRNA and protein levels differed between resistant cells and parental cells. Accordingly, we examined the mechanism by which Smad4 expression affected the response of colorectal cancer cell lines to PT. To this end, we employed a lentivirus to overexpress Smad4 in resistant cells, which decreased the $\mathrm{IC}_{50}$ values of PT in colorectal cancer cell lines. Specifically, PT significantly inhibited the migration and in vivo growth of these tumor cells and promoted their apoptosis, suggesting that Smad4 expression affects the chemosensitivity of colorectal cancer cell lines to PT. Therefore, combined interventions based on Smad4 and PT may produce a better outcome than application of PT alone to PT-resistant colorectal cancer. How does Smad4 mediate this effect? For example, increases in MDR1, an ATP-dependent membrane transport protein involved in drug resistance, reportedly decreased accumulation of anticancer drugs in cells (26). However, we found that Smad4 decreased the levels of this protein. Based on these data, we concluded that in these 3 PT-resistant cell lines, Smad4 overexpression directly downregulated the expression of the the MDR1 protein, and consequently resulted in the accumulation of PT, which inhibited the phosphorylation of NF- $\mathrm{B}$ p 65 and the expression of Bcl-2, and promoted the expression of Bax and caspase-3. All alterations of these proteins associated with cell migration and apoptosis are consistent with previous studies on the mechanism through which PT suppresses tumor cells. Therefore, the overexpression of Smad4 may increase PT accumulation in resistant cells by suppressing MDR1 and significantly decreasing $\mathrm{IC}_{50}$ values in these resistant cell lines. Based on these findings, the following questions warrant further investigation in the near future: Why is Smad4 expression suppressed in PT-resistant colorectal cancer cells? What is the target through which Smad4 restores the sensitivity to PT in resistant colorectal cancer cells? And how does the change in Smad4 expression dynamically affect the content of PT in the resistant cells?

In summary, drug resistance significantly influenced the survival of patients with cancer. The present study revealed that Smad4 re-expression may be crucial for reversing the resistance to PT in PT-resistant colorectal cancer.

\section{References}

1. Sun J, Zhang C, Bao YL, Wu Y, Chen ZL, Yu CL, Huang YX, Sun Y,Zheng LH, Wang X, et al: Parthenolide-induced apoptosis, autophagy and suppression of proliferation in HepG2 cells. Asian Pac J Cancer Prev 15: 4897-4902, 2014.

2. Carlisi D, D'Anneo A, Martinez R, Emanuele S, Buttitta G, Di Fiore R, Vento R, Tesoriere G and Lauricella M: The oxygen radicals involved in the toxicity induced by parthenolide in MDA-MB-231 cells. Oncol Rep 32: 167-172, 2014.

3. Al-Fatlawi AA, Al-Fatlawi AA, Irshad M, Rahisuddin and Ahmad A: Effect of parthenolide on growth and apoptosis regulatory genes of human cancer cell lines. Pharm Biol 53: 104-109, 2015.

4. Lu C, Wang W, Jia Y, Liu X, Tong Z and Li B: Inhibition of AMPK/autophagy potentiates parthenolide-induced apoptosis in human breast cancer cells. J Cell Biochem 115: 1458-1466, 2014.

5. Yu HJ, Jung JY, Jeong JH, Cho SD and Lee JS: Induction of apoptosis by parthenolide in human oral cancer cell lines and tumor xenografts. Oral Oncol 51: 602-609, 2015.

6. Ding Z, Wu CJ, Chu GC, Xiao Y, Ho D, Zhang J, Perry SR, Labrot ES, Wu X, Lis R, et al: SMAD4-dependent barrier constrains prostate cancer growth and metastatic progression. Nature 470: 269-273, 2011. 
7. Chen C, Sun MZ, Liu S, Yeh D, Yu L, Song Y, Gong L, Hao L, $\mathrm{Hu}$ J and Shao S: Smad4 mediates malignant behaviors of human ovarian carcinoma cell through the effect on expressions of E-cadherin, plasminogen activator inhibitor-1 and VEGF. BMB Rep 43: 554-560, 2010.

8. Inamoto S, Itatani Y, Yamamoto T, Minamiguchi S, Hirai H, Iwamoto M, Hasegawa S, Taketo MM, Sakai Y and Kawada K Loss of SMAD4 promotes colorectal cancer progression by accumulation of myeloid-derived suppressor cells through the CCL15-CCR1 chemokine axis. Clin Cancer Res 22: 492-501, 2016.

9. Nikolic A, Ristanovic M, Zivaljevic V, Rankov AD, Radojkovic D and Paunovic I: SMAD4 gene promoter mutations in patients with thyroid tumors. Exp Mol Pathol 99: 100-103, 2015.

10. Boulay JL, Mild G, Lowy A, Reuter J,Lagrange M, Terracciano L, Laffer U, Herrmann R and Rochlitz C: SMAD4 is a predictive marker for 5-fluorouracil-based chemotherapy in patients with colorectal cancer. Br J Cancer 87: 630-634, 2002.

11. Papageorgis P, Cheng K, Ozturk S, Gong Y, Lambert AW, Abdolmaleky HM, Zhou JR and Thiagalingam S: Smad4 inactivation promotes malignancy and drug resistance of colon cancer. Cancer Res 71: 998-1008, 2011.

12. Kim SL, Liu YC, Seo SY, Kim SH, Kim IH, Lee SO, Lee ST, Kim DG and Kim SW: Parthenolide induces apoptosis in colitis-associated colon cancer, inhibiting NF- $\kappa \mathrm{B}$ signaling. Oncol Lett 9: 2135-2142, 2015.

13. Zhao X, Liu X and Su L: Parthenolide induces apoptosis via TNFRSF10B and PMAIP1 pathways in human lung cancer cells. J Exp Clin Cancer Res 33: 3, 2014.

14. Ghantous A, Saikali M, Rau T, Gali-Muhtasib H, Schneider-Stock R and Darwiche N: Inhibition of tumor promotion by parthenolide: Epigenetic modulation of $p 21$. Cancer Prev Res 5: 1298-1309, 2012.

15. Li CJ, Guo SF and Shi TM: Culture supernatants of breast cancer cell line MDA-MB-231 treated with parthenolide inhibit the proliferation, migration, and lumen formation capacity of human umbilical vein endothelial cells. Chin Med J 125: 2195-2199, 2012.

16. Sohma I, Fujiwara Y, Sugita Y, Yoshioka A, Shirakawa M, Moon JH, Takiguchi S, Miyata $\mathrm{H}$, Yamasaki M, Mori M, et al: Parthenolide, an NF- $\kappa$ B inhibitor, suppresses tumor growth and enhances response to chemotherapy in gastric cancer. Cancer Genomics Proteomics 8: 39-47, 2011.
17. Xin Y, Yin F, Qi S, Shen L, Xu Y, Luo L, Lan L and Yin Z: Parthenolide reverses doxorubicin resistance in human lung carcinoma A549 cells by attenuating NF- $\kappa \mathrm{B}$ activation and HSP70 up-regulation. Toxicol Lett 221: 73-82, 2013.

18. Liu D, Liu Y, Liu M, Ran L and Li Y: Reversing resistance of multidrug-resistant hepatic carcinoma cells with parthenolide. Future Oncol 9: 595-604, 2013.

19. Ramachandra M, Atencio I, Rahman A, Vaillancourt M, Zou A, Avanzini J, Wills K, Bookstein R and Shabram P: Restoration of transforming growth factor Beta signaling by functional expression of smad4 induces anoikis. Cancer Res 62: 6045-6051, 2002.

20. Li X, Liu B, Xiao J, Yuan Y, Ma J and Zhang Y: Roles of VEGF-C and Smad4 in the lymphangiogenesis, lymphatic metastasis, and prognosis in colon cancer. J Gastrointest Surg 15: 2001-2010, 2011.

21. Bierie B and Moses HL: Tumour microenvironment: TGFbeta: the molecular Jekyll and Hyde of cancer. Nat Rev Cancer 6: 506-520, 2006

22. Kozak MM, von Eyben R, Pai J, Vossler SR, Limaye M, Jayachandran P, Anderson EM, Shaffer JL, Longacre T, Pai RK, et al: Smad4 inactivation predicts for worse prognosis and response to fluorouracil-based treatment in colorectal cancer. J Clin Pathol 68: 341-345, 2015.

23. Alhopuro P, Alazzouzi H, Sammalkorpi H, Dávalos V, Salovaara R, Hemminki A, Järvinen H, Mecklin JP, Schwartz S Jr, Aaltonen LA, et al: SMAD4 levels and response to 5-fluorouracil in colorectal cancer. Clin Cancer Res 11: 6311-6316, 2005.

24. Zhang B, Leng C, Wu C, Zhang Z, Dou L, Luo X, Zhang B and Chen X: Smad4 sensitizes colorectal cancer to 5-fluorouracil through cell cycle arrest by inhibiting the PI3K/Akt/ CDC2/survivin cascade. Oncol Rep 35: 1807-1815, 2016.

25. Yu SL, Lee DC, Son JW, Park CG, Lee HY and Kang J: Histone deacetylase 4 mediates SMAD family member 4 deacetylation and induces 5-fluorouracil resistance in breast cancer cells. Oncol Rep 30: 1293-1300, 2013.

26. Abdallah HM, Al-Abd AM, El-Dine RS and El-Halawany AM: P-glycoprotein inhibitors of natural origin as potential tumor chemo-sensitizers: A review. J Adv Res 6: 45-62, 2015. 